\title{
Establishment and application of a multiple cross displacement amplification combined with nanoparticles-based biosensor method for the detection of Bordetella pertussis
}

Shijun $\mathrm{Li}^{1+}$, Chunting Liu ${ }^{1+}$, Ying Liu', Qing Ma ${ }^{1}$, Yue Wang ${ }^{1}$ and Yi Wang ${ }^{2,3^{*}}$

\begin{abstract}
Background: Bordetella pertussis is the causative agent of pertussis, a respiratory tract infectious disease. Efficient techniques for detection of B. pertussis isolates are important for clinical diagnosis. Multiple cross displacement amplification (MCDA), a novel isothermal amplification based molecular detection method, has been developed to overcome the technical drawback of the current methods in recent years. This aim of this study is to develop a MCDA with Nanoparticles-based Lateral Flow Biosensor (MCDA-LFB) for the detection of B. pertussis.

Results: A set of 10 primers based on the pertussis toxin (PT) promoter region sequence of $B$. pertussis was designed. The $B$. pertussis-MCDA-LFB assay was successfully established and optimized at $64^{\circ} \mathrm{C}$ for reaction of $40 \mathrm{~min}$. The detection limit was determined as $10 \mathrm{fg} /$ reaction of pure DNA, and no cross-reactions to non-B. pertussis strains were observed, based on the specificity validation. The whole operation, ranging from template preparation to result reporting, could be completed within 70 min without requirement of costly equipment. The B. pertussis-MCDA-LFB in clinic sample detection yielded identical positive rates with traditional culture and showed higher sensitivity than conventional PCR. The results of MCDA-LFB are easier to read due to the usage of LFB.

Conclusions: The isothermal amplification based MCDA-LFB established in the present study is a specific, sensitive, rapid and economical technique for the detection of B. pertussis.
\end{abstract}

Keywords: Bordetella pertussis, Multiple cross displacement amplification, Lateral flow biosensor, MCDA- LFB, Detection limit

\footnotetext{
* Correspondence: wildwolf0101@163.com

tShijun Li and Chunting Liu contributed equally to this work.

${ }^{2}$ Department of Respiratory Disease, Beijing Pediatric Research Institute,

Beijing Children's Hospital, Capital Medical University, National Center for

Children's Health, Beijing 10045, PR China

${ }^{3}$ Key Laboratory of Major Diseases in Children, Ministry of Education, Beijing

Key Laboratory of Pediatric Respiratory Infection Disease, National Clinical

Research Center for Respiratory Diseases, Beijing Children's Hospital, Capital

Medical University, National Center for Children's Health, Beijing 10045, PR

China

Full list of author information is available at the end of the article
}

(c) The Author(s). 2020 Open Access This article is licensed under a Creative Commons Attribution 4.0 International License, which permits use, sharing, adaptation, distribution and reproduction in any medium or format, as long as you give appropriate credit to the original author(s) and the source, provide a link to the Creative Commons licence, and indicate if changes were made. The images or other third party material in this article are included in the article's Creative Commons licence, unless indicated otherwise in a credit line to the material. If material is not included in the article's Creative Commons licence and your intended use is not permitted by statutory regulation or exceeds the permitted use, you will need to obtain permission directly from the copyright holder. To view a copy of this licence, visit http://creativecommons.org/licenses/by/4.0/ The Creative Commons Public Domain Dedication waiver (http://creativecommons.org/publicdomain/zero/1.0/) applies to the data made available in this article, unless otherwise stated in a credit line to the data. 


\section{Background}

Pertussis, also referred whooping cough or named as "cough of 100 days", is a respiratory tract infectious disease primarily leaded by the causative agent Bordetella pertussis [1], a gram negative bacterium which can attach to the ciliated cells and colonize the human upper respiratory tract [2]. It can damage the respiratory epithelium by producing multiple toxins and leads to systemic effects such as the lymphocytosis [3], local inflammatory changes in the mucosal lining of the respiratory tract [1]. Whooping cough can be life threatening for newborns and young children without vaccination. $B$. pertussis can also infect other population such as adults, and a shift of infection cases from schoolage children to adolescents, adults and infants has been observed in the last decade [2, 3]. Although the efficacious pertussis vaccines has been widely used, whooping cough results in about 200,000 deaths annually and at least 24 million new pertussis cases were reported in 2014 in children under 5 years old [2]. Most deaths caused by pertussis are from developing countries, with majority of cases in children under the age of 5 months [4]. According to the estimation of WHO in 2013, B. pertussis infection caused about 60,257 deaths in children younger than 5 years [5].

The appropriate use of clinically accurate diagnostic tests is essential for the effective diagnosis of pertussis. Pertussis can be diagnosed by the commonly used culture and serological methods [6]. However, 7 to 10 days are needed to isolate and identify, B. pertussis isolates using conventional culture method, while the indirect serological diagnosis needs about 1 month based on the reaction with sera, acute- and convalescent-phase sera [7]. As a result of the shortage of culture and serologic diagnosis, the detection results are usually not available even till the patient has already recovered from the illness. In order to improve the detection duration and sensitiveness of laboratory diagnosis, PCR-based methods have been used for the detection of $B$. pertussis. Most available diagnostic PCR assays, including nested PCR and real-time PCR, merely developed based on fewer targets, which cannot differentiate $B$. pertussis from other Bordetella spp. including $B$. holmesii [8]. For example, real time-PCR based on part of insertion sequences (IS) cannot be used for specific species detection, because IS481 also exists in the genome of some B. bronchiseptica strains and B. holmesii strains while IS1001 is proved in some B. bronchiseptica and $B$. parapertussis (Martini, et al., 2017). Other targets including sequence of $\mathrm{BP} 283, \mathrm{BP} 485, p \mathrm{x} \mathrm{S} 1$ and the pertactin genes have been used for the detection of $B$. pertusis, but cross-reactivity with other Bordetella spp. have been reported [9]. It has been reported that the pertussis toxin (PT) promoter sequences differ from the sequence of $B$. pertussis, B. parapertussis and B. bronchiseptica [10, 11], which enable the feasibility to develop PCR based methods targeting the PT promoter region for the specific detection of B. pertusiss. Previous study reported that PCR based on the PT promoter sequence specifically recognize $B$. pertussis, but the sensitivity is insufficient $[12,13]$. More recently, loop-mediated isothermal amplification (LAMP) targeting the PT promoter region has been established to amplify the DNA of $B$. pertussis with high degree of simplicity and specificity [7]. Although LAMP assays showed high efficiency of amplification samples with marginal amounts of DNA were still difficult to detect [14]. Particularly, the results of LAMP techniques for $B$. pertusiss detection were determined using agarose gel electrophoresis, real-time turbidity equipment or color indicator. The operation of gel electrophoresis for the analysis of products is complicated, and the risk of DNA product degradation and carryover contamination is increased [15]. The reading of real-time turbidity of $B$. pertussis-LAMP amplification requires optical instrument, and is easily to be interfered by the background. The judgment of result of B. pertussi-LAMP using the naked eyes is possibly subjective, which is also possible that the result reading be ambiguous to the naked eye due to the low concentration of DNA template.

In recent years, multiple cross displacement amplification (MCDA), a novel isothermal amplification based molecular detection method, has been developed to overcome the technical drawback of the current methods $[16,17]$. MCDA designed ten primers (two displacement primers, two core primers and six amplification primers), instead of two in PCR and six in loop-mediated isothermal amplification assay, to recognize 10 different regions of target sequence, which enhance its sensitivity, specificity, and shorten its reaction time. Moreover, MCDA assay requires merely isothermal conditions and simple equipment such as water bath or heater. The amplification products can be detected by using disposable lateral flow biosensors, with visual result judgment [18], which is objective and does not need any instrument for result reading. MCDA has been validated for the effective detection of various bacteria such as Klebsiella pneumonia, Staphylococcus aureus and Pseudomonas aeruginosa [16, 17]. However, MCDA-LFB has not been validated for the detection of $B$. pertussis. Herein, MCDA targeting the PT promoter region, combined with nanoparticles-based biosensor, was established and optimized for simple, rapid, high specific and sensitive detection of $B$. pertussis at the current report, which was further evaluated by comparison with traditional culture and PCR methods.

\section{Results}

\section{Confirming the effectiveness of $B$. pertussis-MCDA primer} set

To confirm the effectiveness of $B$. pertussis-MCDA primer set targeting on the PT promoter region (Fig. 1, Table 1), the DNA extracted from $B$. pertussis strain was 


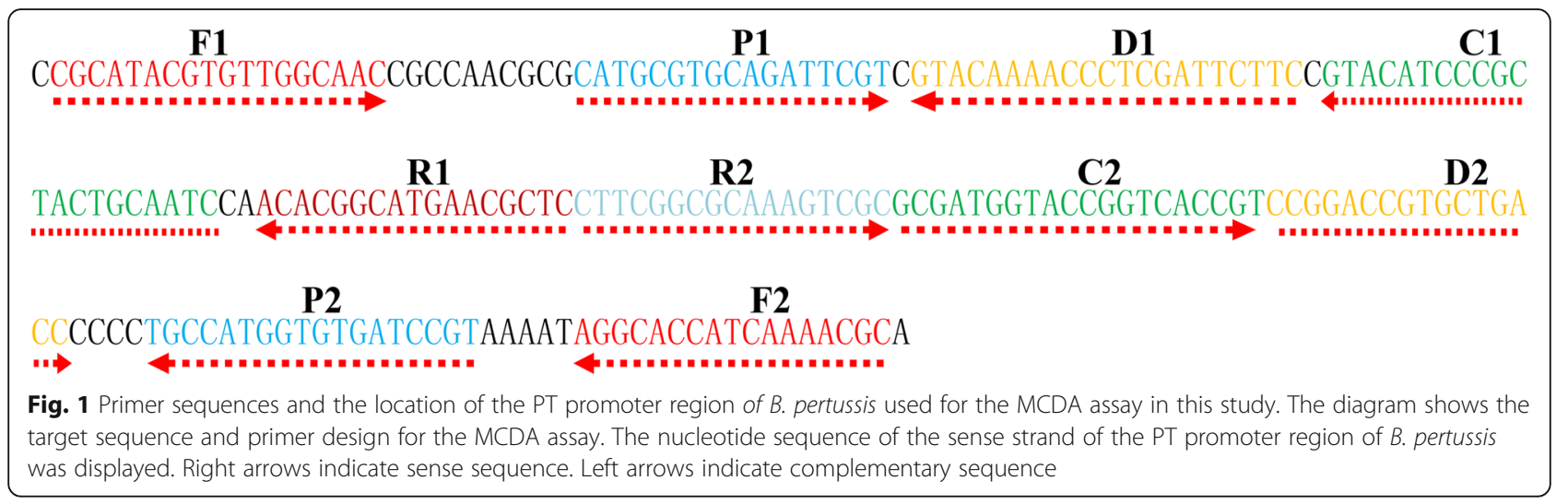

amplified with MCDA at $64^{\circ} \mathrm{C}$ for $1 \mathrm{~h}$. Both the colorimetric indicator (MG) and LFB showed that DNA of $B$. pertussis strain ATCC-9340 was amplified effectively, but no amplification of DNA products was observed for $S$. aureus (GZCDC isolate), $N$. meningitidis (GZCDC isolate) and DW (blank control) (Fig. 2a and b). The electrophoresis gels images, showing a DNA ladder, were observed in positive reaction, but no DNA ladder was observed in the negative and blank controls (Fig. 2c). Therefore, the MCDA primer set was selected as the candidate to establish MCDA-LFB assay for the detection of B. pertussis.

\section{Optimizing the reaction temperature of $B$. pertussis- MCDA-LFB}

To optimize amplification temperature, genomic DNA templates from $B$. pertussis ATCC-9340 were applied as the positive control with $10 \mathrm{pg}$ DNA for each reaction. The real-time turbidity of amplification products was monitored. Typical kinetics graphs were observed for all the determined temperatures (from 60 to $67^{\circ} \mathrm{C}$ with increments of $1{ }^{\circ} \mathrm{C}$ ), and faster amplification was achieved at $64{ }^{\circ} \mathrm{C}$ (Fig. 3). Therefore, $64^{\circ} \mathrm{C}$ was selected as the amplification temperature for the further experiments.

\section{Sensitivity of MCDA-LFB for B. pertussis detection}

Serially diluted genomic DNA from $B$. pertussis was used to examine the detection limit of the $B$. pertussisMCDA-LFB. The results detected with LFB showed that the sensitivity of the B. pertussis-MCDA-LFB was as low as $10 \mathrm{fg}$ (2.4 copies) per reaction (Fig. 4a). By MG, the detection limit of the $B$. pertussis-MCDA assay was also $10 \mathrm{fg}$ per reaction (Fig. 4b), which was in accordance with the detection results of LFB.

\section{Optimal reaction time of $B$. pertussis-MCDA-LFB}

In order to optimize the reaction time of $B$. pertussisMCDA-LFB assay, different time span (10, 20, 30 and 40 min) were chosen for amplification at $64^{\circ} \mathrm{C}$ based on the standard conditions of MCDA. The lowest DNA level (10 fg of B. pertussis DNA templates per reaction) showed both TL and CL lines when the reaction only continued

Table 1 The primers used in this study

\begin{tabular}{|c|c|c|c|}
\hline Primers name ${ }^{a}$ & Sequences and modifications ${ }^{\mathbf{b}}$ & Length $^{c}$ & Gene \\
\hline $\mathrm{F} 1$ & 5'- CGCATACGTGTTGGCAAC-3' & $18 \mathrm{nt}$ & PT promoter region \\
\hline F2 & 5'-GCGTTTGATGGTGCCT-3' & $17 \mathrm{nt}$ & \\
\hline CP1 & 5'-GATTGCAGTAGCGGGATGTACCATGCGTGCAGATTCGT -3' & 38 mer & \\
\hline CP2 & 5'-GCGATGGTACCGGTCACCGTACGGATCACACCATGGC-3' & 37 mer & \\
\hline C1 & 5'-GATTGCAGTAGCGGGATGTAC-3' & $21 \mathrm{nt}$ & \\
\hline $\mathrm{C} 1^{*}$ & 5'-Biotin-GATTGCAGTAGCGGGATGTAC-3' & $21 \mathrm{nt}$ & \\
\hline $\mathrm{C} 2$ & 5'-GCGATGGTACCGGTCACCGT-3' & $20 \mathrm{nt}$ & \\
\hline D1 & 5'-GAAGAATCGAGGGTTITGTAC-3' & $21 \mathrm{nt}$ & \\
\hline D2 & 5'-CCGGACCGTGCTGACC-3' & $16 \mathrm{nt}$ & \\
\hline R1 & 5'-GAGCGTTCATGCCGTGT-3' & $17 \mathrm{nt}$ & \\
\hline $\mathrm{R} 1^{*}$ & 5'-FITC-GAGCGTTCATGCCGTGT-3' & $17 \mathrm{nt}$ & \\
\hline R2 & 5'-CTTCGGCGCAAAGTCGC-3' & $17 \mathrm{nt}$ & \\
\hline
\end{tabular}

${ }^{a} \mathrm{C} 1 *, 5^{\prime}$-labeled with biotin when used in MCDA-LFB assay; $1^{*}{ }^{*}, 5^{\prime}$-labeled with FITC when used in MCDA-LFB assay. ${ }^{\mathrm{b}}$ FITC fluorescein isothiocyanate, ${ }^{\mathrm{c}}$ mer monomeric unit, $n t$ nucleotide, $P T$ pertussis toxin 

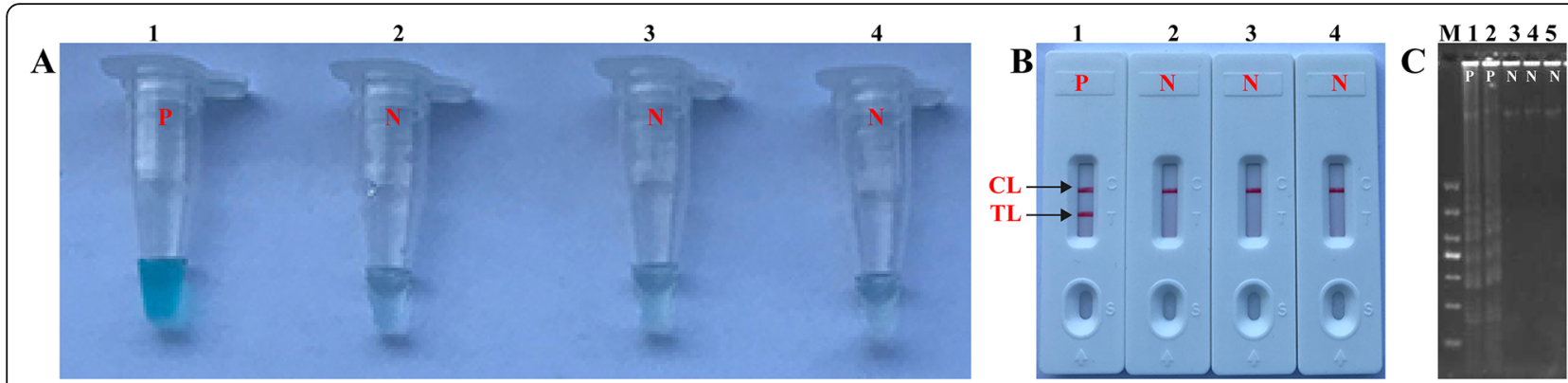

Fig. 2 Effectiveness of B. pertussis-MCDA primer set. a Products of the B. pertussis-MCDA amplification were visually monitored by determination of the color change using the malachite green. $\mathbf{b}$ visual detection of $B$. pertussis-MCDA products using lateral flow biosensor. Tube 1/Biosensor 1: positive results of B. pertussis strain ATCC-9340; Tube 2/Biosensor 2: negative results of S. aureus; Tube 3/Biosensor 3: negative results of N. meningitidis; Tube 4/Biosensor 4: DW (blank control). TL: test line; CL: control line. $\mathbf{c}$ The target-specific formation of MCDA products was confirmed by electrophoresis gels images. M: DNA Marker; 1 and 2: positive results of B. pertussis strain ATCC-9340; 3: negative results of S. aureus; 4: negative results of N. meningitides; 5: DW (blank control)

for $40 \mathrm{~min}$ at temperature of $64^{\circ} \mathrm{C}$. Therefore, 40 min was selected as the optimal amplification time for B. pertussisMCDA-LFB (Fig. 5).

\section{Specificity of B. pertussis-MCDA-LFB assay}

The initial specificity of the MCDA primers for $B$. pertussis detection was confirmed by blast analysis in NCBI data base and the PT promoter sequence were only found in $B$. pertussis strains, with similarity of $99.5 \% \sim 100 \%$. Then, genomic DNA template from $B$. pertussis and non- $B$. pertussis strains (Table 2) were used to assess the specificity of $B$. pertussis-MCDA-LFB Assay. The results showed that two red lines appeared at the location of TL and CL on the strips for the B. pertussis strain, but only one line appeared at the location of $\mathrm{CL}$ for all the non-B. pertussis strains and blank control (Fig. 6), suggesting negative results for non- $B$. pertussis bacterial isolates and DW sample (blank control).

\section{Application of $B$. pertussis-MCDA-LFB in clinical samples} In order to evaluate the $B$. pertussis-MCDA-LFB for clinical application, 67 nasal swab samples were used for DNA template preparation, which were were applied for the evaluation of B. pertussis-MCDA-LFB in clinical application. The detection results demonstrated 31 samples were $B$. pertussis positive when using RT-PCR for detection, while the other 36 samples were proved negative. MCDA-LFB detection demonstrated 37 positive samples, which included the 31 positive samples detected with real-time PCR. Besides, MCDA-LFB showed consistent results with traditional cultivation and isolation methods in the clinic sample detection, both of which confirmed 37 positive clinic samples (Table 3 ).

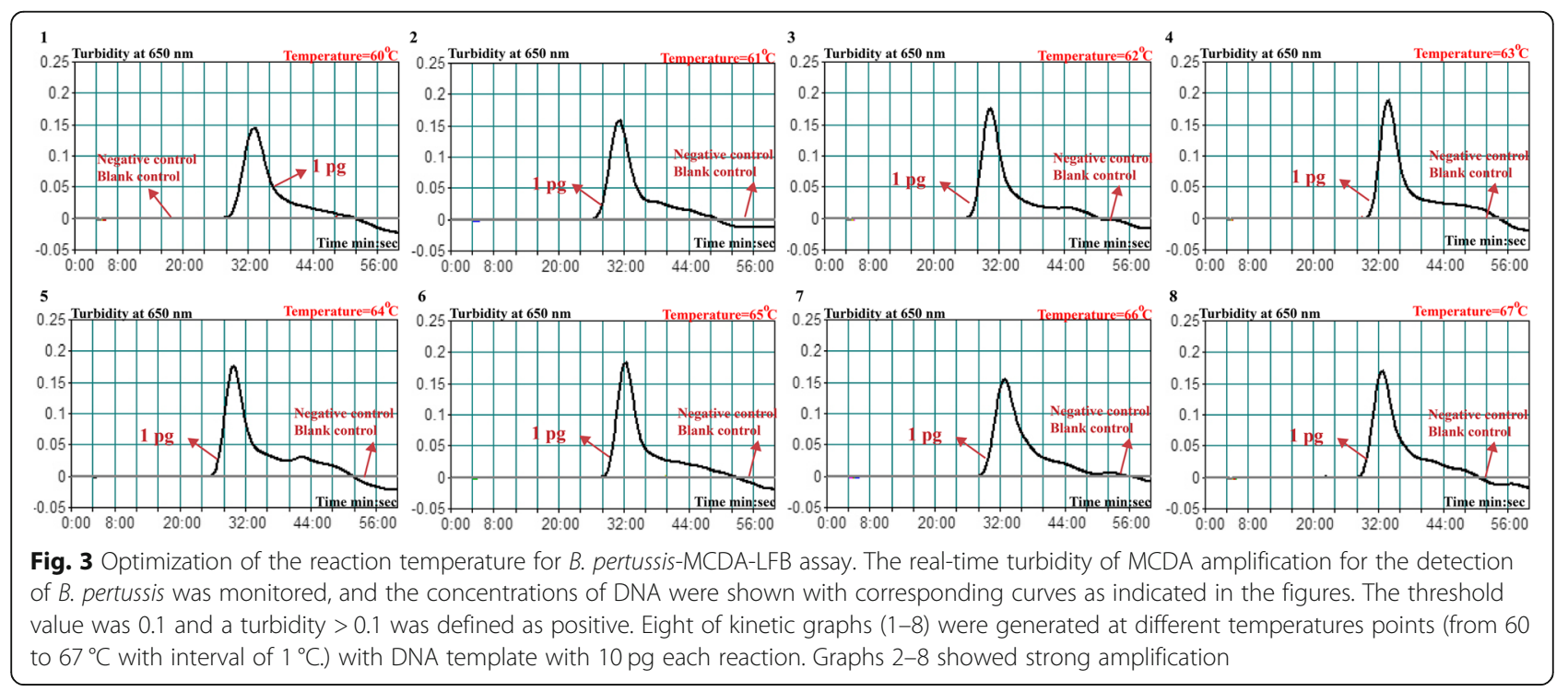




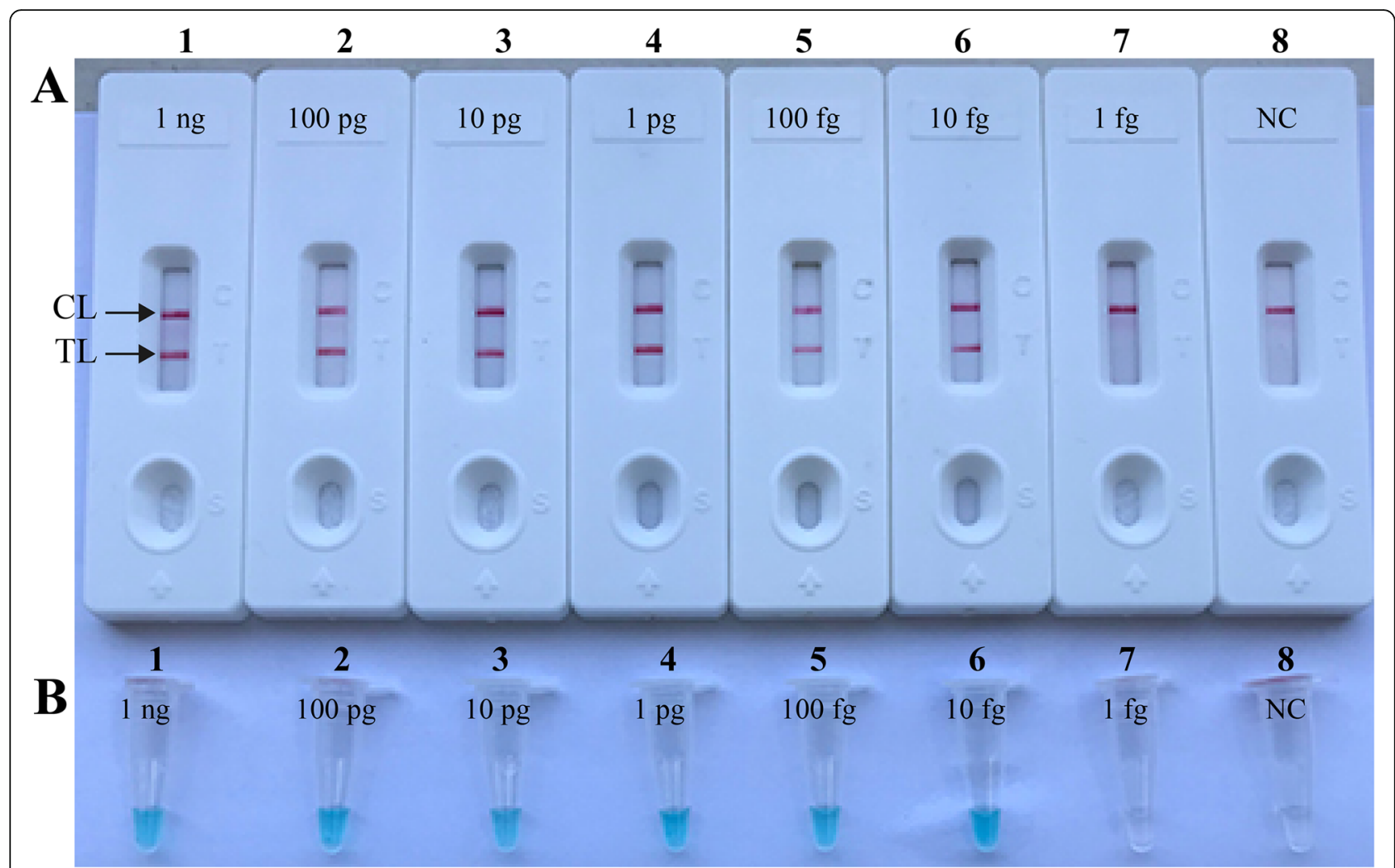

Fig. 4 Sensitivity evaluation of B. pertussis-MCDA-LFB assay for the detection of DNA from B. pertussis strain ATCC-9340. Lateral flow biosensor (a) and colorimetric indicator (b) were used to monitor the reaction products. Biosensors (a)/Tubes (b) from 1 to 7 indicate $1 \mathrm{ng}, 100$ pg, 10 pg, 1 pg, $100 \mathrm{fg}, 10 \mathrm{fg}$, and $1 \mathrm{fg}$ of target DNA; Biosensor 8 indicate DW (negative control), respectively. Amplification of $1 \mathrm{ng}, 100 \mathrm{pg}, 10 \mathrm{pg}, 1 \mathrm{pg}, 100 \mathrm{fg}$ and $10 \mathrm{fg}$ of DNA template showed positive amplification, while the $1 \mathrm{fg}$ per reaction and blank control was negative. NC: negative control; TL: test line; CL: control line

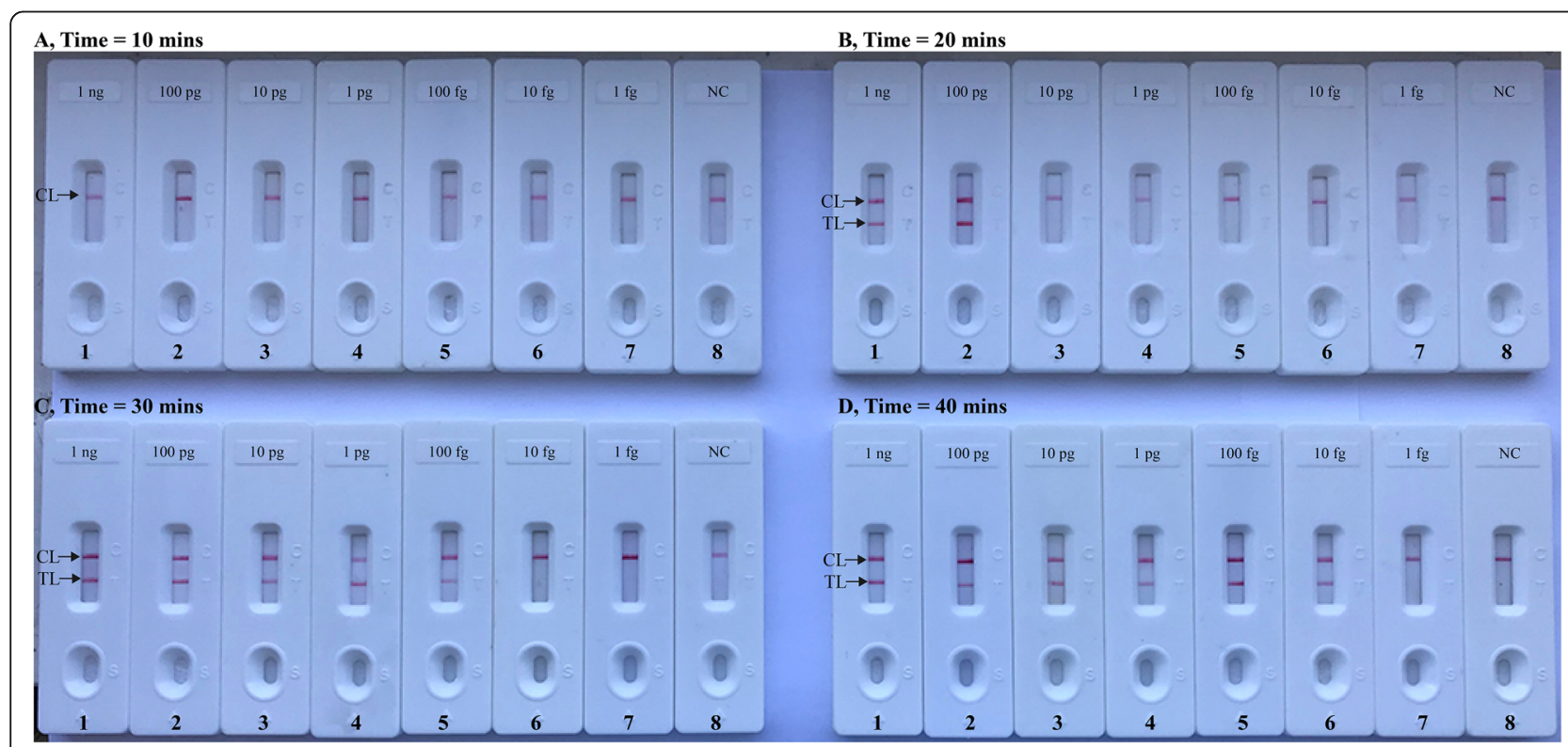

Fig. 5 The optimal reaction time for B. pertussis-MCDA-LFB detection. Four different reaction times including $10 \mathrm{~min}(\mathbf{a}), 20 \mathrm{~min}(\mathbf{b}), 30 \mathrm{~min}$ (c), and $40 \mathrm{~min}$ (d) were examined and compared at $64^{\circ} \mathrm{C}$. Biosensors $1-7$ indicate $1 \mathrm{ng}, 100 \mathrm{pg}, 10 \mathrm{pg}, 1 \mathrm{pg}, 100 \mathrm{fg}, 10 \mathrm{fg}, 1 \mathrm{fg}$ of DNA extracted from B. pertussis, respectively; Biosensor 8 indicates DW (blank control), The optimal sensitivity appeared at 40 min of amplification (c). TL indicates test line; $\mathrm{CL}$ indicates control line 
Table 2 Bacterial strains used in this study

\begin{tabular}{|c|c|c|c|c|}
\hline No. & Bacteria & $\begin{array}{l}\text { Serogroup/ } \\
\text { Species }\end{array}$ & Strain name. (source of strain) ${ }^{a}$ & No. of strains \\
\hline 1 & Bordetella pertussis & $U$ & ATCC9340 & 1 \\
\hline 2 & Bordetella pertussis & $U$ & Isolated strain (GZDC) & 16 \\
\hline 3 & Bordetella parapertussis & $U$ & Isolated strains (GZDC) & 2 \\
\hline 4 & Neisseria meningiditis & $U$ & Isolated strains (GZDC) & 1 \\
\hline 5 & Hemophililus parainfluenza & $U$ & Isolated strains (GZDC) & 1 \\
\hline 6 & Streptococcus pneumoniae & $u$ & Isolated strains (GZDC) & 1 \\
\hline 7 & Klebsiella pneumoniae & $u$ & Isolated strains (GZDC) & 1 \\
\hline 8 & Pseudomonas aeruginosa & $U$ & Isolated strains (GZDC) & 1 \\
\hline 9 & Mycoplasma pneumoniae & U & Isolated strains (GZDC) & 1 \\
\hline 10 & Legionellae bacillus & $u$ & Isolated strains (GZDC) & 1 \\
\hline 11 & Acinetbacter baumannii & $U$ & Isolated strains (GZDC) & 1 \\
\hline 12 & Staphylococcus aureus & $u$ & Isolated strains (GZDC) & 1 \\
\hline 13 & Staphylococcus saprophyticus & $U$ & Isolated strains (GZDC) & 1 \\
\hline 14 & Salmonella & Typhimurium & Isolated strains (GZDC) & 1 \\
\hline 15 & enteropathogenic E. coli & U & Isolated strains (GZDC) & 1 \\
\hline 16 & enterotoxigenic $E$. coli & $U$ & Isolated strains (GZDC) & 1 \\
\hline 17 & invasive E.coli & U & Isolated strains (GZDC) & 1 \\
\hline 21 & enterohemorrhagic E. coli & $U$ & EDL933 & 1 \\
\hline 18 & enteroaggregative E.coli & $U$ & Isolated strains (GZDC) & 1 \\
\hline 19 & Streptococcus suis & $U$ & Isolated strains (GZDC) & 1 \\
\hline 20 & Vibrio cholerae & U & Isolated strains (GZDC) & 1 \\
\hline 21 & Enterococcus faecalis, & $U$ & ATCC35667 & 1 \\
\hline 22 & Enterococcus faecium & U & Isolated strains (GZDC) & 1 \\
\hline 23 & Bacillus proteus & $U$ & Isolated strains (GZDC) & 1 \\
\hline 24 & Enterobacter cloacae & U & Isolated strains (GZDC) & 1 \\
\hline 25 & Listeria monocytogenes, & $4 a$ & ATCC19114 & 1 \\
\hline 26 & Shigella flexneri, & F1a & Isolated strains (GZDC) & 1 \\
\hline 27 & Shigella boydii & $U$ & Isolated strains (GZDC) & 1 \\
\hline
\end{tabular}

${ }^{a} U$ unidentified serotype, ATCC American Type Culture Collection

\section{Discussion}

It has been reported that 156 million cases of pneumonia each year in children younger than 5 years are estimated by the World Health Organization (WHO) [19, 20]. Recent evidence shows a sizable infant cases of pertussis present with acute pneumonia and $2 \%$ of clinical pneumonia cases of enrolled infants were caused by pertussis $[20,21]$. B. pertussis is the major causative reagent of pertussis, albeit other Bordetella bacteria such as $B$. parapertussis and B. holmesii [22] can cause less severe symptoms. Accurate and timely diagnosis of pertussis is extremely important, but the diagnosis of pertussis and accurate laboratory detection of Bordetella infections is still challenging. For instance, the commonly used culture of $B$. pertussis is fastidious, with limited sensitivity [23], while previously developed PCR-based methods of
B. pertussis have been confirmed with the shortage of low sensitivity or cross-reaction with other Bordetella bacteria strains $[8,9,12,13]$.

In this study, MCDA-LFB based on the PT promoter region of $B$. pertussis, with excellent specificity, was successfully established. The high specificity of $B$. pertussisMCDA-LFB is likely due to applying PT promoter region as detection target $[12,13]$. Furthermore, the primer set based on different sequence of the promoter region also contributed the high degree of specificity of B. pertussis-MCDA, which has been previously demonstrated [24]. Particularly, the MCDA primer set, which recognized 10 regions of $\mathrm{PT}$ promoter, also ensured the assay's specificity. Although several closely related species (such as B. bronchiseptica, B. holmessii and B. hinzii) did not be examined to validate B. pertussis-MCDA- 


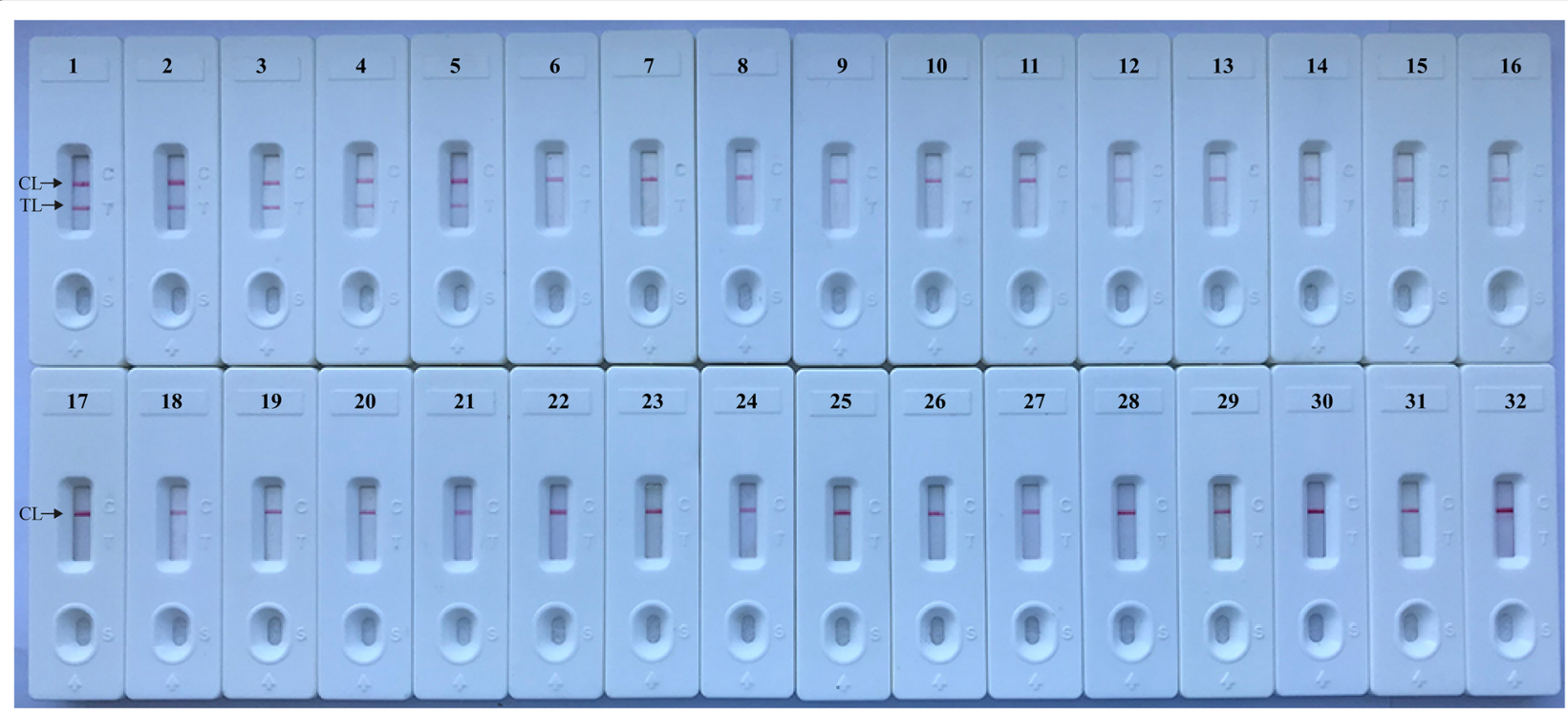

Fig. 6 Specificity assessment of the B. pertussis-MCDA-LFB Assay. Different genomic DNA templates were applied for B. pertussis-MCDA-LFB specificity evaluation. Biosensor 1 used DNA template from B. pertussis strain ATCC-9340; Biosensors 2-5 used DNA templates from B. pertussis strain (GZCDC isolate), respectively. Biosensor 6 used DNA template from Bordetella parapertussis. Biosensors 7-31 used DNA template from bacterial strain of Neisseria meningiditis, Hemophililus parainfluenza, Streptococcus pneumoniae, Klebsiella pneumoniae, Pseudomonas aeruginosa, Mycoplasma pneumoniae, Legionellae bacillus, Acinetbacter baumannii, Staphylococcus aureus, Staphylococcus saprophyticus, Salmonella, enteropathogenic E. coli, enterotoxigenic E. coli, invasive E.coli, enterohemorrhagic E. coli, enteroaggregative E.coli, Streptococcus suis, Vibrio cholerae, Enterococcus faecalis, Enterococcus faecium, Bacillus proteus, Enterobacter cloacae; Listeria monocytogenes, Shigella flexneri, Shigella boydii, respectively. Biosensor 32 used DW as negative control

LFB's specificity, the data of specificity from NCBI BLAST has revealed that the MCDA primer set designed here was specific to $B$. pertussis. Furthermore, the specificity of the assay was further evaluated by using non- $B$. pertussis strains (Fig. 6) and clinical samples (Table 3). Therefore, the B. pertussis-MCDA-LFB established in the present study is of high degree of specificity.

Except the advantage of specificity, the $B$. pertussisMCDA-LFB established in the present study also displayed excellent sensitivity. The detection limit for $B$. pertussis pure cultures reached as low as $10 \mathrm{fg}$ DNA per reaction (Fig. 4). Compared with the official real-time PCR assay recommended by China CDC, the B. pertussis-MCDA-LFB displayed better sensitivity because the limit of detection of real-time PCR kit was 70 copies per reaction (equivalent to $292 \mathrm{fg}$ per reaction) provided by

Table 3 Comparison of real-time PCR, culture-biotechnical and MCDA-LFB for the detection of Bordetella pertussis in clinical samples

\begin{tabular}{lll}
\hline Detection & samples $(n=67)$ & \\
\cline { 2 - 3 } methods & Positive & Negative \\
\hline Real-time PCR ${ }^{\text {a }}$ & 31 & 36 \\
Culture & 37 & 30 \\
MCDA-LFB & 37 & 30 \\
\hline
\end{tabular}

${ }^{a}$ The real-time PCR was designed using the PT promoter region of B. pertussis its manual. Furthermore, the application of detection for $B$. pertussis in clinical samples also verified the outstanding sensitivity of the $B$. pertussis-MCDA-LFB, which yielded higher positive rates than real-time PCR assay (Table 3). The lower detection rate of real-time PCR may be due to the factors that the copy numbers of the B. pertussis templates were lower than LoD (limit of detection), or the presence of some inhibitors specific to real-time PCR decreased the reaction sensitivity.

Fast diagnosis of the pathogenic agents contributes to the accurate clinical diagnosis and rational therapy of patients. Traditional culture method needs more than 1 week to determine $B$. pertussis in the clinical sample, while serological diagnosis needs about 1 month [7, 25]. MCDA-LFB assay developed in this study can be finished within $70 \mathrm{~min}$, which includes $25 \mathrm{~min}$ for sample preparation, $40 \mathrm{~min}$ for amplification reaction and $2 \mathrm{~min}$ of results judgment. Lateral flow biosensor used for result interpretation in this study is time-saving, simpler compared with other methods such as traditional culture, PCR based methods or LAMP. Besides, result interpretation with LFB is based on the test line and control line, thus it is more objective and less error-prone compared with traditional culture, PCR based methods or LAMP.

Detection cost may be an economic burden for many laboratories, particularly in developing countries, which may influence the timely diagnosis for patients. The $B$. 
pertussis-MCDA-LFB merely needs a simple incubation at $64^{\circ} \mathrm{C}$, because MCDA-LFB is independent of thermal denature and temperature changes during the reaction. Therefore, it has extensive application prospects in resource-poor laboratories, which lack the advanced and precise equipment. Besides, each MCDA-LFB test only costs \$5.5 USD, including bout \$3.5 USD for reaction and \$2 USD for LFB. Additionally, Certified technical personnel are not the prerequisite for the operation of MCDA-LFB due to its easy and simple operation, so that the labor costs are decreased. Therefore, B. pertussisMCDA-LFB assay exhibited distinct strength in terms of costs for equipment, labor, experimental reagents and material. Notably, our B. pertussis-MCDA-LFB assay used similar sample collection and template extraction techniques as the B. pertussis-PCR and RT-PCR assays, it is subject to the potential shortcomings with regard to the availability of extraction kits, extraction instruments and reagents.

\section{Conclusions}

$B$. pertussis-MCDA-LFB was successfully developed for the effective detection of $B$. pertussis. It exhibited strength of specificity for the detection of $B$. pertussis, with detection limit of $10 \mathrm{fg}$ of DNA from pure culture of $B$. pertussis. It can be finished within $60 \mathrm{~min}$, with convenient and simple operation and it does not require costly equipment. Thus, the $B$. pertussis-MCDA-LFB assay offers an effective strategy for rapid detection of $B$. pertussis, which is of potential application value in the field and resource-poor laboratories.

\section{Methods}

\section{Reagents and instruments}

The reagents of Malachite Green (MG) and Isothermal Amplification Kits were ordered from BeilingHaiTaiZhengYuan Technology Co., Ltd. (Beijing, China). DNA Extraction Kits were purchased from SBS Genetech Co., Ltd. (Beijing, China). Crimson red and streptavidin coated polymer nanoparticles were obtained from Bangs Laboratories, INC. (Indiana, USA). Biotinylated bovine serum albumin (Biotin-BSA) and rabbit antifluorescein antibody (Anti-FITC Ab) were obtained from the Abcam Co., Ltd. (Shanghai, China). Nitrocellulose (NC) membranes, membrane backing materials, sample pads, conjugate pads and absorbent pads were produced by the Jie Yi Biotechnology Co., Ltd. (Shanghai, China).

\section{Nanoparticle-based biosensor}

Lateral flow biosensor (LFB, $4 \mathrm{~mm} \times 60 \mathrm{~mm}$ ) used in this study was prepared according to previously reported operation with some modifications [26]. Briefly, a backing card was laminated with a sample pad, absorbent pad conjugate pad and NC membrane. The test line (TL) and control line (CL) were prepared by spraying $\mathrm{NC}$ membrane with anti-FITC Ab $(0.25 \mathrm{mg} / \mathrm{ml})$ and biotinBSA $(2.5 \mathrm{mg} / \mathrm{ml})$, with separation of $5 \mathrm{~mm}$ between the TL and CL. The conjugate pad of the strip was then coated with streptavidin which was coated with polymer nanoparticles resolved in $0.01 \mathrm{M} \mathrm{PBS}$ (PH 7.4). The prepared cards were sliced into 4-mm-wide strips (Deli No. 8012) and packed with plastic bag accompanied with desiccant gel and stored at RT (room temperature).

\section{Genomic DNA template preparation}

B. pertussis strains were cultured on charcoal agar plates (OXOID, UK) supplemented with15\% of defibrinated sheep blood at $37^{\circ} \mathrm{C}$ for 3 to 5 days [27]. DNA Extraction Kit (SBS Genetech, Beijing, China) were used to prepare the DNA template based on the operation instruction. DNA was quantified with the instrument Nano drop ND1000 (Calibre, Beijing, China). $100 \mathrm{ng} / \mu \mathrm{L}$ of target DNA was used for a 10 -fold dilution $(1 \mathrm{ng} / \mu \mathrm{L}, 100 \mathrm{pg} / \mu \mathrm{L}, 10 \mathrm{pg} /$ $\mu \mathrm{L}, 1 \mathrm{pg} / \mu \mathrm{L}, 100 \mathrm{fg} / \mu \mathrm{L}, 10 \mathrm{fg} / \mu \mathrm{L}$ and $1 \mathrm{fg} / \mu \mathrm{L}$ ), which were equivalent to $2.4 \times 10^{5}$ copies $/ \mu \mathrm{L}, 2.4 \times 10^{4}$ copies $/ \mu \mathrm{L}$, $2.4 \times 10^{3}$ copies $/ \mu \mathrm{L}, 2.4 \times 10^{2}$ copies $/ \mu \mathrm{L}, 2.4 \times 10^{1}$ copies $/$ $\mu \mathrm{L}, 2.4 \times 10^{0}$ copies $/ \mu \mathrm{L}$ and $2.4 \times 10^{-1}$ copies $/ \mu \mathrm{L}$. In particular, the number of genomic copies for each dilution was confirmed based on the fact that $4.17 \mathrm{fg}$ of genomic template was equivalent to a single genome of $B$. pertussis (assuming a molecular size of $4.1 \mathrm{Mbp}$ for $B$. pertussis) $[28,29]$. The serial dilution of genomic DNA of $B$. pertussis strain ATCC-9340 was used to test the sensitivity of $B$. pertussis-MCDA-LFB. Non- $B$. pertussis strains were mixed with $10 \%(\mathrm{w} / \mathrm{v})$ glycerol broth and kept at $-70{ }^{\circ} \mathrm{C}$. The genomic DNA of non-B. pertussis strains (Table 2) were extracted with the QIAamp DNA Mini Kit (Qiagen, Germantown, MD, USA).

\section{B. pertussis-MCDA primer set design and synthesis}

The PT promoter sequence targeting $B$. pertussis was applied for MCDA primer design. A set of 10 primers (F1, F2, CP1, CP2, C1, C1*, C2, D1, D2, R1* and R2) was designed by using PREMIER 5.0 to establish MCDA assay. Integrated DNA Technologies design tools were applied to analyze the hairpin structures and hybrids of primer sequences. Specificity of the MCDA primers for $B$. pertussis detection was confirmed by blast analysis in NCBI data base. The biotin and fluorescein isothiocyanate (FITC) was used to label the $5^{\prime}$ ends of $\mathrm{C} 1$ and R1 primer, individually. The primer information is shown in Fig. 1 and Table 1. All the primers of HPLC purification grade were produced by Tianyi-Huiyuan Biotech Co., Ltd. (Beijing, China).

\section{The B. pertussis-MCDA reaction}

MCDA reactions were performed in $25 \mu \mathrm{l}$ reaction system as previously described [30]. The reaction system 
for each sample contained $12.5 \mu$ l of $2 \times$ reaction mix (isothermal amplification ${ }^{\bullet}$ kit), $0.4 \mu \mathrm{M}$ of primer $\mathrm{F} 1$ and $\mathrm{F} 2,0.8 \mu \mathrm{M}$ of primer $\mathrm{C} 1{ }^{*}, \mathrm{C} 2, \mathrm{R} 1 *$, R2, D1 and D2, $1.6 \mu \mathrm{M}$ of cross primer $\mathrm{CP} 1$ and $\mathrm{CP} 2,1 \mu \mathrm{l}$ of Bst 2.0 DNA polymerase $(8 \mathrm{U})$ and $1 \mu \mathrm{l}$ of DNA template. MG and LFB detection were simultaneously applied to monitor the MCDA amplification products. By using the MG method, color changes from colorless to light green as a result of the reaction products should be detectable while no color changes observed in the negative and blank control. When using the LFB for product detection, two visible lines should be observed in positive reactions at the location of $\mathrm{CL}$ and $\mathrm{TL}$, respectively, but only one line at the location of CL appear in the blank controls and negative. By confirming the target-specific formation of MCDA products by electrophoresis gels images, a DNA ladder should be observed in positive reaction, but no DNA ladder observed in the negative and blank control.

\section{Optimizing the amplification temperature of $B$. pertussis- MCDA-LFB}

Real-time turbidity determination was used to monitor the MCDA reactions for detecting the DNA B. pertussis. The optimum reaction temperature was determined using DNA of $\mathrm{B}$. pertussis from $60^{\circ} \mathrm{C}$ to $67^{\circ} \mathrm{C}$ for 60 min, with interval of $1^{\circ} \mathrm{C}$. Turbidimeter (LA-320C) was used to monitor the turbidity of MCDA amplification. Genomic DNA $(1 \mu \mathrm{l})$ of $S$. aureus and $N$. meningitidis strains were used as negative controls, and doubledistilled water (DW) were chosen as blank controls.

\section{B. pertussis-MCDA-LFB sensitivity determination}

The serial dilutions of of B. pertussis genomic templates described above were used to determine the limit detection of B. pertussis-MCDA assay. LFB and colorimetric indicator (MG) were used to detect the MCDA amplification results, respectively.

\section{Optimizing the amplification time of $B$. pertussis-MCDA- LFB}

The serially diluted DNA was used to optimize the MCDA reaction time. MCDA reaction mixture was incubated for $10,20,30$ or $40 \mathrm{~min}$ at the optimal temperature. The MCDA amplification products were detected with a LFB, Test of each amplification time were repeated at least two times.

\section{B. pertussis-MCDA-LFB specificity determination}

The genomic DNA templates of $17 \mathrm{~B}$. pertussis strains and 27 non-B. pertussis strains were applied to validate the specificity of $B$. pertussis-MCDA. LFB was used to detect the amplification results (Table 2). The examinations for MCDA specificity validation were repeated at least two times.

\section{Application of the $B$. pertussis-MCDA-LFB in clinical samples}

A total of 67 nasal swab samples, collected from patients distributed in the prefecture Guiyang, Anshun, Zunyi, Tongren, Qiannan, Qiandongnan Qianxinan and Bijie of Guizhou Province, were used for the clinical validation of $B$. pertussis-MCDA-LFB. Particularly, the clinical samples used in this study, which were part of the routine CDC (Center for Disease Control and Prevention) laboratory procedure in China, were not specifically isolated for this research. The National Health and Family Planning Commission of China determined that the collection of data from human cases of infectious disease was part of continuing public health surveillance of a notifiable infectious disease and was exempt from institutional review board assessment [31]. All data were supplied and analyzed in an anonymous format, without access to personal identifying information.

The total genomic DNA templates from the clinical samples stored in $-70{ }^{\circ} \mathrm{C}$ for further use after regular detection by traditional culture and a real-time PCR assay (Recommended by China CDC) were used for the evaluation of $B$. pertussis-MCDA-LFB in clinic detection. The specificity and sensitivity of $B$. pertussis-MCDALFB, traditional culture and the conventional real-time PCR for the detection of the genomic DNA of B. pertussis from the clinical samples were compared. In particular, the $B$. pertussis-RT-PCR assay recommended by China CDC (MABSKY Biotech Co., Ltd., Beijing, China) was conducted according its manual ("SKY-PCR-FZ300-1). Briefly, each $25 \mu \mathrm{L}$ reaction mix contained $12.5 \mu \mathrm{L}$ of $2 \times$ Reaction Mix, $2 \mu \mathrm{L}$ of primer mix, $5.5 \mu \mathrm{L}$ of deionized water and $5 \mu \mathrm{L}$ of templates. Amplification was carried out as the following conditions: $95^{\circ} \mathrm{C}$ for 3 mins, followed by 40 cycles of $95^{\circ} \mathrm{C}$ for $5 \mathrm{~s}, 55^{\circ} \mathrm{C}$ for 40 s.

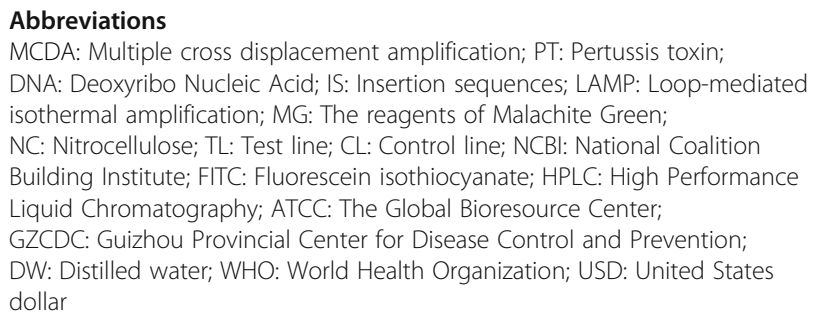

Acknowledgements

Not applicable.

Authors' contributions

YiW and SL conceived and designed the experiments. SL, CL, YL, QM and YuW conducted the experiments. YiW and SL analyzed the data. SL and YiW contributed the reagents, materials, analysis tools. YiW operated the 
software. YiW and SL prepared the manuscript. All authors have read and approved the manuscript.

\section{Funding}

This study was funded by the Program of High-Level Creative Talents Cultivation in Guizhou Province (Qian Ke He (2016)4021), Program of Scientific and Technological Innovation Team of Guizhou Province (Qian Ke He Platform talent [2018]5606) and Program of Academician workstation of Guizhou Province (Qian Ke He Platform talent [2018]5627). The funding body had no role in the design of the study, data collection and analysis, interpretation of data, or in writing the manuscript.

\section{Availability of data and materials}

The datasets supporting our findings are included in the article.

\section{Ethics approval and consent to participate}

These clinical samples used in this study were not specifically isolated for this research. They were part of the routine CDC (Center for Disease Control and Prevention) laboratory procedure in China. The National Health and Family Planning Commission of China determined that the collection of data from human cases of infectious disease was part of continuing public health surveillance of a notifiable infectious disease and was exempt from institutional review board assessment. All data were supplied and analyzed in an anonymous format, without access to personal identifying information. Therefore, the need for consent was deemed unnecessary.

\section{Consent for publication}

Not applicable.

\section{Competing interests}

All the authors listed in the paper declare that they have no competing interests for publication of this paper.

\section{Author details}

'Laboratory of Bacterial Infectious Disease of Experimental Center, Guizhou Provincial Center for Disease Control and Prevention, Guiyang 550004, Guizhou, China. ${ }^{2}$ Department of Respiratory Disease, Beijing Pediatric Research Institute, Beijing Children's Hospital, Capital Medical University, National Center for Children's Health, Beijing 10045, PR China. ${ }^{3}$ Key Laboratory of Major Diseases in Children, Ministry of Education, Beijing Key Laboratory of Pediatric Respiratory Infection Disease, National Clinical Research Center for Respiratory Diseases, Beijing Children's Hospital, Capital Medical University, National Center for Children's Health, Beijing 10045, PR China.

Received: 17 December 2019 Accepted: 12 August 2020 Published online: 24 August 2020

\section{References}

1. Lauria AM, Zabbo CP. Pertussis (Whooping Cough). In: StatPearls. Treasure Island: StatPearls Publishing; 2019.

2. Solans $\mathrm{L}$, Locht $\mathrm{C}$. The role of mucosal immunity in pertussis. Front Immunol. 2018;9:3068

3. Gall SA. Prevention of pertussis, tetanus, and diphtheria among pregnant, postpartum women, and infants. Clin Obstet Gynecol. 2012;55(2):498-509.

4. Winter K, Zipprich J, Harriman K, Murray EL, Gornbein J, Hammer SJ, Yeganeh N, Adachi K, Cherry JD. Risk factors associated with infant deaths from pertussis: a case-control study. Clin Infect Dis. 2015:61(7):1099-106.

5. Yeung KHT, Duclos P, Nelson EAS, Hutubessy RCW. An update of the global burden of pertussis in children younger than 5 years: a modelling study. Lancet Infect Dis. 2017;17(9):974-80

6. Lee AD, Cassiday PK, Pawloski LC, Tatti KM, Martin MD, Briere EC, Tondella ML, Martin SW, Group CVS. Clinical evaluation and validation of laboratory methods for the diagnosis of Bordetella pertussis infection: Culture, polymerase chain reaction (PCR) and anti-pertussis toxin lgG serology (lgGPT). PLoS One. 2018;13(4):e0195979.

7. Kamachi K, Toyoizumi-Ajisaka H, Toda K, Soeung SC, Sarath S, Nareth Y, Horiuchi Y, Kojima K, Takahashi M, Arakawa Y. Development and evaluation of a loop-mediated isothermal amplification method for rapid diagnosis of Bordetella pertussis infection. J Clin Microbiol. 2006;44(5):1899-902.
8. Martini H, Detemmerman L, Soetens O, Yusuf E, Pierard D. Improving specificity of Bordetella pertussis detection using a four target real-time PCR. PLoS One. 2017;12(4):e0175587.

9. Probert WS, Ely J, Schrader K, Atwell J, Nossoff A, Kwan S. Identification and evaluation of new target sequences for specific detection of Bordetella pertussis by real-time PCR. J Clin Microbiol. 2008;46(10):3228-31.

10. Nygren M, Reizenstein E, Ronaghi M, Lundeberg J. Polymorphism in the pertussis toxin promoter region affecting the DNA-based diagnosis of Bordetella infection. J Clin Microbiol. 2000;38(1):55-60.

11. Arico B, Rappuoli R. Bordetella parapertussis and Bordetella bronchiseptica contain transcriptionally silent pertussis toxin genes. J Bacteriol. 1987;169(6): 2847-53.

12. Grimprel E, Begue P, Anjak I, Betsou F, Guiso N. Comparison of polymerase chain reaction, culture, and western immunoblot serology for diagnosis of Bordetella pertussis infection. J Clin Microbiol. 1993;31(10):2745-50.

13. Houard S, Hackel C, Herzog A, Bollen A. Specific identification of Bordetella pertussis by the polymerase chain reaction. Res Microbiol. 1989;140(7):47787.

14. Wang Y, Zhang L, Liu D, Luo L, Li H, Cao X, Liu K, Xu J, Ye C. Multiplex, rapid, and sensitive isothermal detection of nucleic-acid sequence by endonuclease restriction-mediated real-time multiple cross displacement amplification. Front Microbiol. 2016;7:753.

15. Zhu X, Wang X, Han L, Chen T, Wang L, Li H, Li S, He L, Fu X, Chen S. Multiplex reverse transcription loop-mediated isothermal amplification combined with nanoparticle-based lateral flow biosensor for the diagnosis of COVID-19. Biosens Bioelectron. 2020;166:112437.

16. Wang Y, Yan W, Fu S, Hu S, Xu J, Ye C. Multiple cross displacement amplification coupled with nanoparticles-based lateral flow biosensor for detection of staphylococcus aureus and identification of methicillinResistant S aureus. Front Microbiol. 2018:9:907.

17. Zhao F, Niu L, Nong J, Wang C, Wang J, Liu Y, Gao N, Zhu X, Wu L, Hu S. Rapid and sensitive detection of Pseudomonas aeruginosa using multiple cross displacement amplification and gold nanoparticle-based lateral flow biosensor visualization. FEMS Microbiol Lett. 2018;365(14):fny147.

18. Wang Y, Li H, Li D, Li K, Xu J, Ye C. Multiple cross displacement amplification combined with gold nanoparticle-based lateral flow biosensor for detection of Vibrio parahaemolyticus. Front Microbiol. 2016;7:2047.

19. Rudan I, Boschi-Pinto C, Biloglav Z, Mulholland K, Campbell H. Epidemiology and etiology of childhood pneumonia. Bull World Health Organ. 2008;86(5): 408-16.

20. El Basha NR, Shaaban HH, El Atroush HA, Sherif MM, El Kholy AA. The use of multiplex PCR for the detection of atypical pathogens in Egyptian children with CAP: a high rate of Bordetella pertussis in early infancy. J Egypt Public Health Assoc. 2019;94(1):5.

21. Zar HJ, Barnett W, Stadler A, Gardner-Lubbe S, Myer L, Nicol MP. Aetiology of childhood pneumonia in a well vaccinated south African birth cohort: a nested case-control study of the Drakenstein child health study. Lancet Respir Med. 2016;4(6):463-72.

22. Mazengia E, Silva EA, Peppe JA, Timperi R, George H. Recovery of Bordetella holmesii from patients with pertussis-like symptoms: use of pulsed-field gel electrophoresis to characterize circulating strains. J Clin Microbiol. 2000; 38(6):2330-3.

23. Zouari A, Smaoui $H$, Kechrid A. The diagnosis of pertussis: which method to choose? Crit Rev Microbiol. 2012;38(2):111-21.

24. Wang $Y$, Wang $Y$, Lan $R, X u$ H, Ma A, Li D, Dai H, Yuan X, Xu J, Ye C. Multiple endonuclease restriction real-time loop-mediated isothermal amplification: a novel analytically rapid, sensitive, multiplex loop-mediated isothermal amplification detection technique. J Mol Diagn. 2015;17(4):392-401.

25. Mattoo S, Cherry JD. Molecular pathogenesis, epidemiology, and clinical manifestations of respiratory infections due to Bordetella pertussis and other Bordetella subspecies. Clin Microbiol Rev. 2005;18(2):326-82.

26. Wang $Y, L i ~ H$, Wang $Y, X u H, X u J$, Ye C. Antarctic thermolabile uracil-DNAglycosylase-supplemented multiple cross displacement amplification using a label-based nanoparticle lateral flow biosensor for the simultaneous detection of nucleic acid sequences and elimination of carryover contamination. Nano Res. 2018;11(5):2632-47.

27. Liu W, Xu Y, Dong D, Li H, Zhao X, Li L, Zhang Y, Wei X, Wang X, Huang S, et al. Survey and rapid detection of Bordetella pertussis in clinical samples targeting the BP485 in China. Front Public Health. 2015;3:39.

28. Parkhill J, Sebaihia M, Preston A, Murphy LD, Thomson N, Harris DE, Holden MT, Churcher CM, Bentley SD, Mungall KL. Comparative analysis of the 
genome sequences of Bordetella pertussis, Bordetella parapertussis and Bordetella bronchiseptica. Nat Genet. 2003;35(1):32-40.

29. Nakamura Y, Kamachi K, Toyoizumi-Ajisaka H, Otsuka N, Saito R, Tsuruoka J, Katsuta T, Nakajima N, Okada K, Kato T. Marked difference between adults and children in Bordetella pertussis DNA load in nasopharyngeal swabs. Clin Microbiol Infect. 2011;17(3):365-70.

30. Wang Y, Wang Y, Ma AJ, Li DX, Luo L, Liu DX, Jin D, Liu K, Ye CY. Rapid and sensitive isothermal detection of nucleic-acid sequence by multiple cross displacement amplification. Sci Rep. 2015;5:11902.

31. Li S, Liu C, Liu Y, Ma Q, Wang Y, Wang Y. Development of a multiple cross displacement amplification combined with nanoparticles-based biosensor assay to detect Neisseria meningitidis. Infect Drug Resist. 2019;12:2077.

\section{Publisher's Note}

Springer Nature remains neutral with regard to jurisdictional claims in published maps and institutional affiliations.

Ready to submit your research? Choose BMC and benefit from:

- fast, convenient online submission

- thorough peer review by experienced researchers in your field

- rapid publication on acceptance

- support for research data, including large and complex data types

- gold Open Access which fosters wider collaboration and increased citations

- maximum visibility for your research: over $100 \mathrm{M}$ website views per year

At BMC, research is always in progress.

Learn more biomedcentral.com/submissions 